\title{
DEVELOPING A DRIVER-CENTRIC ROADWAY CLASSIFICATION SYSTEM WITH MULTIDIMENSIONAL SCALING
}

\author{
Stacy Balk, Vaughan Inman, \& William Perez \\ Science Applications International Corporation (SAIC), Transportation Solutions Division \\ McLean, Virginia, USA \\ Email: Stacy.Balk.ctr@dot.gov
}

\begin{abstract}
Summary: Various systems exist to classify roadway environments; however most do not consider driver-relevant perceptual components. A perceptually based roadway classification system has the potential to support the placement of signage (or removal of extraneous clutter) in the right-of-way as a means to enhance driver performance. The present study sought to determine which environmental factors are attended to by roadway users. Thirteen participants first rated the similarity of 14 roadway environments and then rated each environment on 5 different descriptors (built-up, clutter, openness, aesthetically pleasing, organized/predictable). The resultant data were analyzed using a methodology rarely taken advantage of in the field of transportation: Multidimensional Scaling (MDS). MDS revealed the participants relied on two primary dimensions when rating the similarity of the roadway environments. These two dimensions related closely with: 1) organization/predictability and 2) clutter and aesthetics. This methodology provides a simple way to gain access to drivers' perceptions of the roadway environment and appears to be a promising first step toward developing a user-focused roadway classification system.
\end{abstract}

\section{INTRODUCTION}

Classifying the roadway environment is an important, yet challenging task. There have been many attempts to accomplish this task and classify our visual environment in a meaningful way (e.g., Gibson, 1979; Li, Socher, \& Fei-Fei, 2009). For instance, the Federal Highway Administration employs a functional use system (i.e., interstate, other freeways, principal arterials, minor arterials, major collectors, minor collectors, and local roads; FHWA, 1989). This system relies on road functionality yet ignores other environmental characteristics. As an example, arterial roads in urban and rural areas can look vastly different. An arterial in a suburban/rural area may consist of two travel lanes and be surrounded by foliage and minimal signage. On the other hand, an arterial in an urban environment may contain six travel lanes and be surrounded by buildings and ample commercial signage. Alternative roadway classification systems may rely on situational components - items that move freely to and from the area (e.g., pedestrians, vehicles, etc.). Items in a scene can also be classified as built, or permanent, components (e.g., grocery stores, trees, etc.). Whereas these types of functional use/component classification systems are quite useful in some respects (e.g., the implementation of speed limits, curbing, sidewalks, etc.), they are unable to provide substantial objective information about environmental influences that may be vital to driver performance (e.g., speed limit signs, stop signs, construction zone warnings, miscellaneous visual clutter, etc.). Furthermore, these classifications fail to capture many driver-relevant perceptual differences between environments. 
Driver-relevant perceptual components of an environment are important for many reasons. As an example, Goldenbeld and van Schagen (2007) found that roadway/roadside characteristics (curvature, clarity of situation, vegetation right side of the road, and road width) significantly influence drivers' perceptions of safe travel speeds. These types of perceptual differences (cues and affordances) can be exploited in roadway design. One manner in which this can be done is through self-explaining roadways (SER). SERs are roads that utilize/modify visual characteristics of the environment (e.g., speed humps, road width, building setback distance, etc.) to influence driver behavior and minimize the severity of crashes. As an example, Charlton, Mackie, Baas, Hay, Menezes, and Dixon (2010) assessed speed on urban roads before and after the implementation of SER principles. It was found that the changes in roadway environment both reduced overall speed and increased speed homogeneity (i.e., curtailing extreme speed differences between vehicles) - outcomes that are desirable along many roads.

Speed selection is not the only area where environmental perceptual cues can play an influential role in driver performance. For example, it has been shown that visually demanding tasks can lead drivers to reduce vehicle speeds (Antin, Dingus, Hulse, \& Wierwille, 1990; Curry, Hieatt, \& Wilde, 1975; Engström, Johansson, \& Östlund, 2005). Vehicle slowing/stopping may result in traffic flow delays, resulting in both monetary costs and time losses (e.g., Schrank \& Lomax, 2009; Komanoff \& Fisher, 2010). Furthermore, this seemingly erratic behavior (i.e., speed variability) could potentially lead to crashes, near misses, and frustration among fellow road users. It is difficult however, to determine exactly what constitutes a high visual load, or what tasks are too visually demanding - after all, we live in an extraordinarily visually rich environment.

Thus it is difficult to determine how much information in the environment is too much. When a visual scene or array is discussed, clutter is often mentioned (e.g., Shontz, Trumm, \& Williams, 1971; Yamani \& McCarley, 2010). In this context, clutter, refers to items (or areas) in the visual field-of-view that hinder performance on a specific search task. That is to say, clutter is task and environment dependent. As such, items considered to be clutter (visually distracting) in one scenario, may be extraneous in another. For example, imagine an especially messy (physical) desktop. Assume your task is to locate a phone number printed on a loose sheet of paper. All non-paper (or non-paper-like) items are likely to be immediately discarded from the search. On the other hand, if your goal is to locate the keyboard, most (or all) of the items that were previously considered clutter or distractors (i.e., paper) are now simply extraneous. This, however, does not necessarily indicate that the other non-clutter objects are inconsequential or irrelevant.

In general, when performing a visual search task (looking for a specific target item among other items), the more non-target items present the longer it takes to complete the task (e.g., Treisman, 1986; Bravo \& Farid, 2004; McPhee, Scialfa, Dennis, Ho, \& Caird, 2004). However, the exact manner in which we count these items is debatable (e.g., a person could be counted as a single item or as multiple items if shirt, shoes, pants, nose, fingers, etc., are considered separate elements). Further, the number of non-target items (set size) alone does not dictate the amount of time required to fixate upon and identify a specific target object (in many cases the target item “pops out” - e.g., a red line among black lines; see Johnson \& Proctor, 2004 for overview). People also have a tendency to direct attention toward items of relevance (e.g., Treisman, 1982), 
or "guide" attention to specific task-relevant areas of a visual environment (e.g., Wolfe, 1994). This bias, in the context of the previous desktop example, implies that while the non-relevant items on the desk may increase the time it takes to locate the target object, the non-relevant items themselves may not capture visual attention.

These visual search phenomena have been shown to operate in a driving environment. Drivers tend to fixate on task relevant signage. However, it may be the case that in especially visually complex environments, excessive clutter may increase the time required to identify, to interpret, and to respond to task specific stimuli. This delay may lead to unsafe driving behavior (e.g., McPhee, et al., 2004). It is easy to imagine requiring a relatively long amount of time to identify (or even completely miss) a relevant sign (e.g., lane ending) among a plethora of other signs along a busy/high traffic/high sign content street. As a result, the driver may be startled by a sudden change in the roadway or behave unsafely (e.g. sudden stop, “cutting-off” another vehicle, etc.). This is not to say that all non-traffic control signs in the right-of-way are excessive, non-essential, or even dangerous. It should be noted that the relationship between commercial signage near the right-of-way and driver behavior is not clear; while some studies have reported a link between billboards and driver distraction or crashes, others have failed to find such a relationship (see Farbry, Wochinger, Shafer, Owens, \& Nedzesky, 2001 for a review). To a driver in a novel environment, a commercial billboard may pose as clutter when the driver is searching for a speed limit sign. However, the same billboard could act as driving aid when the driver is searching for an otherwise inconspicuous business.

It is obvious that the roadway environment is complex and it can be difficult to determine exactly what visual information degrades "good" driving and what does not. However, it is likely beneficial to increase the saliency of environmental components that improve driving performance. A first step toward accomplishing this goal is to better understand which items drivers attend to in a roadway environment. Once a better understanding of these factors is gained, the information can be exploited in roadway design by directing drivers' attention to pertinent items/information, yet away from non-essential roadway 'clutter.' As a result of the potential to increase safety through driver-centric roadway design, the present study sought to gain a better understanding of drivers' perceptions of the roadway environment. These perceptions will be used in the interpretation of data from a series of studies on driver eye glance behavior, and detection and identification of highway signs. Participants were asked to rate the similarity of various photographs of roadway environments. The analysis of observer perceptions of roadway environments utilized a somewhat novel methodology for the realm of transportation research: Multidimensional Scaling (MDS; e.g., Young, 1985). MDS analyses result in spatial mapping (i.e., X, Y coordinates) of the scene similarity perceptions. Such maps can provide valuable clues as to the how drivers perceive roadway environments. When combined with other studies, this type of information has the potential to extend our understanding of the interactions between roadway sign placement, lane markings, and roadway geometry with other environmental factors that influence driver performance.

\section{METHOD}

Thirteen people (7 males) at a local community center participated (mean age $=39.8$ years, range $=24$ - 63 years); each had a valid driver's license. First, participants were asked to rate the 
similarity of 14 panoramic photographs of roadways (see Figure 1). The pairs were rated on a scale from 1 (not at all similar) to 10 (very similar). Each photograph was paired with each of the other photographs once (for a total of 91 pairs) and appeared as the upper or lower member of a pair an equal number of times. The panoramic photographs (presented at approximately $960 \mathrm{x}$ 304 pixels) consisted of a variety of typical roadway environments: 2 to 4 lanes, divided and undivided, urban and suburban, residential and commercial. After rating the similarity of each of the pairs of photographs, participants were asked to rate each of the roadway photographs (presented individually) on 5 different descriptors (for a total of 70 ratings; 5 for each of the 14 photographs): built-up, clutter, openness, aesthetically pleasing, organized/predictable. The ratings were on a 1-10 scale, e.g., anchors of "very built-up" and "not at all built-up." (Additional descriptions/clarifications of the adjectives were not provided, unless requested by the participant.) It was hoped that these descriptors, which were derived in pilot testing, would map to the dimensions participants attended to when rating the similarity of the roadway environments.

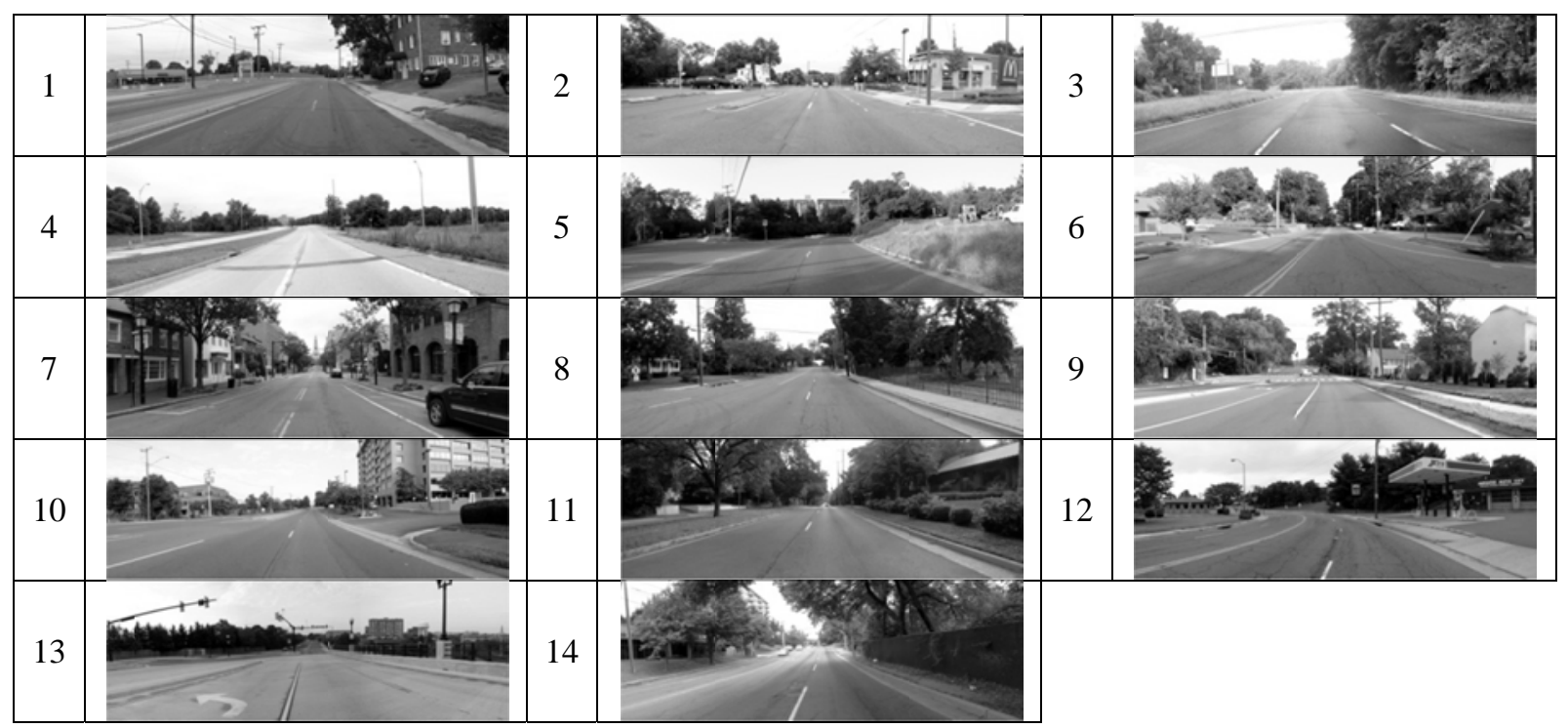

Figure 1. Thumbnail representations of the photographs that participants were asked to rate (The numerical values identify each of the environments in Figure 2)

\section{RESULTS}

The Multidimensional Scaling routine PROXSCAL (SPSS) was used to analyze similarity ratings of the photographs. The data were treated as interval scale. An examination of the scree plot suggested a two dimensional solution (stress $=.117$ ). The two dimensional solution, a mapping of participants' ratings of similarities (and dissimilarities) in two dimensional space, is shown in Figure 2. Dimension 1 is plotted along the $\mathrm{X}$-axis and dimension 2 is plotted along the Y-axis.

Correlations between each of the descriptors and the dimension coordinates were also calculated. As can be seen in Table 1, each of the MDS dimensions was significantly correlated with at least one of the pre-selected descriptors. The first dimension (the X axis of Figure 2) of the MDS solution is significantly correlated with three of the descriptors: built-up $(r=.56, p<.05)$, clutter 
( $r=.75, p<.01)$, and aesthetically pleasing $(r=-.66, p<.01)$. To better understand this dimension, the relationship between the individual descriptors is examined next. The descriptors built-up and clutter are strongly positively correlated with one another $(r=.78, p<.01)$. This relationship suggests, at least in this study, that those environments with a high degree of manmade or built components were also judged to be cluttered. This first dimension was also significantly negatively correlated with pleasing aesthetics, yet this descriptor was not significantly related to any of the others. Thus, this first dimension remains a bit difficult to interpret. It appears that a verbal description of this dimension lies somewhere in a compromise between cluttered and aesthetically pleasing. The second dimension (the Y axis of Figure 2) appears to be related to the predictability or organization of the environment $(r=-.55, p<.05)$. The predictability/organization of an environment has the potential to provide a driver with a great deal of valuable information (e.g., other road user behavior).

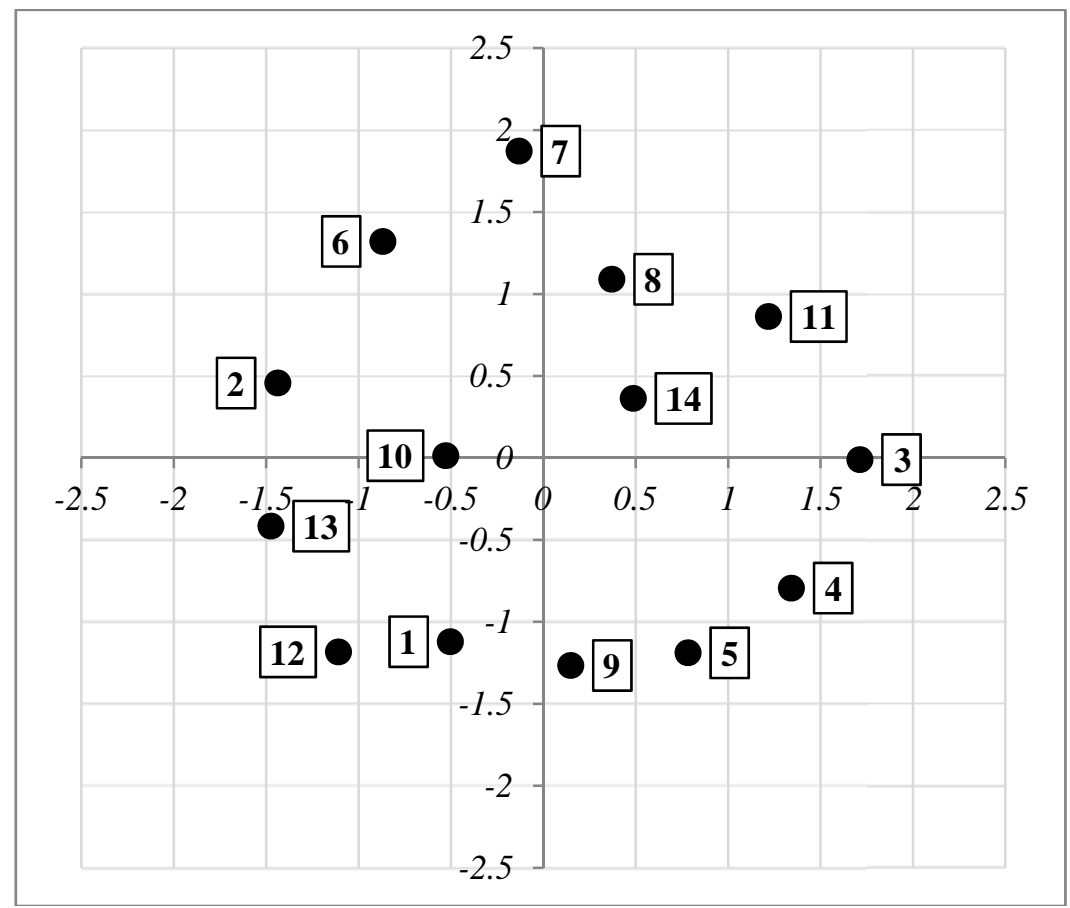

Figure 2. Graphical representation of 2-dimensional MDS solution (The boxed numerical values refer the roadway environments presented in Figure 1)

Table 1. Matrix of correlations between each of the descriptor ratings and each of the MDS dimensions

\begin{tabular}{lcccccc}
\hline & Dimension 2 & Built-up & Clutter & Openness & $\begin{array}{c}\text { Aesthetically } \\
\text { Pleasing }\end{array}$ & $\begin{array}{c}\text { Organized/ } \\
\text { Predictable }\end{array}$ \\
\hline Dimension 1 & -.010 & $.559^{*}$ & $.745^{* *}$ & -.437 & $-.664^{* *}$ & -.472 \\
Dimension 2 & & -.391 & -.108 & .394 & -.485 & $-.554^{*}$ \\
Built-up & & & $.779^{* *}$ & $-.756^{* *}$ & -.128 & -.011 \\
Clutter & & & & $-.691^{* *}$ & -.457 & -.369 \\
Openness & & & & & .074 & .125 \\
Aesthetically & & & & & $.826^{* *}$ \\
\hline
\end{tabular}

*Significant $<.05 ; * *$ Significant $<.01$ 


\section{DISCUSSION}

This study, even with a moderately small sample size, successfully utilized multidimensional scaling to identify environmental factors that observers perceive and attend to when identifying roadway environment similarities. Two major factors, or dimensions, arose. These dimensions mapped fairly well to pre-identified roadway environment descriptors. This mapping provides further insight into the types of characteristics that are salient to observers when examining roadway environments. It appears that participants attended to environmental components that have the potential to affect driving behavior. For example, in an organized/predictable environment one may be able to better predict where vehicles are likely to turn or pedestrians are likely to cross and appropriate reactions to such events may be made more rapidly.

While the MDS methodology is not perfect and is limited in scope, it provides a simple mechanism by which drivers' perceptions of the roadway environment can be assessed. The current study utilized a range of roadway scenes that intentionally did not vary widely in many ways (e.g., roadway traffic was minimal). It is important that subsequent research in this area include a wide variety of roadway environments (e.g., traffic, rural areas, etc.). MDS relies on comparisons of nearly exhaustive pairings between items. As a result, the number of items to be compared generally needs to be limited to around 15 to avoid requiring an overwhelming amount of time from each participant. Nonetheless, MDS may prove to be a robust, easily deployable (e.g., mass internet testing), methodology for identifying aspects of an environment that drivers attend to if the items for comparison are carefully selected. MDS is an observer-centric methodology for examining relationships between stimuli (in this case, roadway environments). As a result, MDS, in combination with other research tools, has the potential to set the foundation for a driver-centric roadway classification system. The application of this type of knowledge base and classification system has the potential to enhance roadway design by using perceptually relevant information to direct drivers' attention to safety enhancing elements and away from those that are extraneous. Furthermore, in combination with necessary on-road behavioral measures, this scaling system has the potential to further guide sign placement regulations (ranging from traffic control devices to off-premise advertisements).

\section{REFERENCES}

Antin, J., Dingus, T., Hulse, M., \& Wierwille, W. (1990). An evaluation of the effectiveness and efficiency of an automobile moving-map navigational display. International Journal of ManMachine Studies, 33, 581-594.

Bravo, M. J., \& Farid, H. (2004). Search for a category target in clutter. Perception, 33, 643-652.

Charlton, S.G., Mackie, H.W., Baas, P.H., Hay, K., Menezes, M., \& Dixon, C. (2010). Using endemic road features to create self-explaining roads and reduce vehicle speeds. Accident Analysis and Prevention, 42, 1989-1998.

Curry, G. A., Hieatt, D. J., and Wilde, G. J. S. Task load in the motor vehicle operator: A comparative study of assessment procedures. Ottawa, Ontario: Ministry of Transport, Road and Motor Vehicle Traffic Safety Branch, 1975.

Engström, J., Johansson, E., \& Östlund, J. (2005). Effects of visual and cognitive load in real and simulated motorway driving. Transportation Research F, 8, 97-120. 
Farbry, J., Wochinger, K., Shafer, T., Owens, N. \& Nedzesky, A. (2001). Research Review of Potential Safety Effects of Electronic Billboards on Driver Attention and Distraction. Federal Highway Administration, Washington, DC.

Gibson, J.J. (1979). The ecological approach to visual perception. Boston: Houghton-Mifflin.

Goldenbeld, C., \& van Schagen, I. (2007). The credibility of speed limits on $80 \mathrm{~km} / \mathrm{h}$ rural roads: The effects of road and person(ality) characteristics. Accident Analysis and Prevention, 39, 1121-1130.

Johnson, A., \& Proctor, R. W. (2004). Attention: Theory and practice. Thousand Oaks, CA: Sage.

Komanoff, C., \& Fisher, W. (2010). Time Thieves: A New Computer-Driven Traffic Model Reveals the "Time Costs" of Traffic. New York Transportation Journal, Articles and Research, 13, Accessed: November 24, 2010 (http://wagner.nyu.edu/rudincenter/journal/2010/02/time-thieves-a-new-computer-driventraffic-model-reveals-the-\%E2\%80\%9Ctime-costs\%E2\%80\%9D-of-traffic/)

Li, L., Socher, R., \& Fei-Fei, L. (2009). Towards total scene understanding: Classification, annotation and segmentation in an automatic framework. Proceedings of International Conference on Computer Vision and Pattern Recognition, 2036-2043.

McPhee, L.C., Scialfa, C.T., Dennis, W.M., Ho, G., and Caird, J.K. (2004). Age differences in visual search for traffic signs during a simulated conversation. Human Factors, 46, 674-685.

Schrank, D., \& Lomax, T. (2009). The 2009 Urban Mobility Report. College Station: Texas Transportation Institute, Texas A\&M University.

Shontz, W.D., Trumm, G.A., \& Williams, L.G. (1971). Color coding for information location. Human Factors, 13, 237-246.

Treisman, A. (1982). Perceptual grouping and attention in visual search for features and for objects. Journal of Experimental Psychology: Human Perception and Performance, 8, 194214.

Treisman, A. (1986). Features and objects in visual processing. Scientific American, 255, 114b125.

US FHWA, 1989. FHWA Functional Classification Guidelines. US Federal Highway Administration, Washington, DC, http://www.fhwa.dot.gov/planning/fctoc.htm.

Wolfe, J.M. (1994) Guided Search 2.0: A Revised Model of Visual Search. Psychonomic Bulletin \& Review, 1, 202-238.

Yamani, Y., and McCarley, J.S. (2010). Visual search asymmetries within color-coded and intensity-coded displays. Journal of Experimental Psychology: Applied, 16, 124-132.

Young, F. (1985). Multidimensional Scaling. In S. Kotz, N. L. Johnson, and C. B. Reads, Eds., Encyclopedia of Statistical Sciences, (pp. 649-659), New York: Wiley. 